\title{
Improved Surface Parameter Retrievals using AIRS/AMSU Data
}

\author{
Joel Susskind $^{1}$ and John Blaisdell ${ }^{2}$ \\ ${ }^{1}$ NASA Goddard Space Flight Center, Greenbelt, MD, USA 20771 \\ ${ }^{2}$ SAIC, NASA Goddard Space Flight Center, Greenbelt, MD, USA 20771
}

\begin{abstract}
The AIRS Science Team Version 5.0 retrieval algorithm became operational at the Goddard DAAC in July 2007 generating near real-time products from analysis of AIRS/AMSU sounding data. This algorithm contains many significant theoretical advances over the AIRS Science Team Version 4.0 retrieval algorithm used previously. Two very significant developments of Version 5 are: 1) the development and implementation of an improved Radiative Transfer Algorithm (RTA) which allows for accurate treatment of non-Local Thermodynamic Equilibrium (non-LTE) effects on shortwave sounding channels; and 2) the development of methodology to obtain very accurate case by case product error estimates which are in turn used for quality control. These theoretical improvements taken together enabled a new methodology to be developed which further improves soundings in partially cloudy conditions. In this methodology, longwave $\mathrm{CO}_{2}$ channel observations in the spectral region $700 \mathrm{~cm}^{-1}$ to $750 \mathrm{~cm}^{-1}$ are used exclusively for cloud clearing purposes, while shortwave $\mathrm{CO}_{2}$ channels in the spectral region $2195 \mathrm{~cm}^{-1}$ to $2395 \mathrm{~cm}^{-1}$ are used for temperature sounding purposes. This allows for accurate temperature soundings under more difficult cloud conditions. This paper further improves on the methodology used in Version 5 to derive surface skin temperature and surface spectral emissivity from AIRS/AMSU observations. Now, following the approach used to improve tropospheric temperature profiles, surface skin temperature is also derived using only shortwave window channels. This produces improved surface parameters, both day and night, compared to what was obtained in Version 5 . These in turn result in improved boundary layer temperatures and retrieved total $\mathrm{O}_{3}$ burden.
\end{abstract}

Keywords: High spectral resolution IR sounders, atmospheric sounding, satellite meteorology, new theoretical developments

\section{INTRODUCTION}

AIRS was launched on EOS Aqua on May 4, 2002, together with AMSU-A and HSB, to form a next generation polar orbiting infrared and microwave atmospheric sounding system. ${ }^{1}$ The primary products of AIRS/AMSU-A are twice daily global fields of atmospheric temperature-humidity profiles, ozone profiles, sea/land surface skin temperature, and cloud related parameters including OLR. Also included are the clear column radiances $\hat{R}_{\mathrm{i}}$ used to derive these products, which are representative of the radiances AIRS would have seen if there were no clouds in the field of view. All products also have error estimates. The sounding goals of AIRS are to produce $1 \mathrm{~km}$ tropospheric layer mean temperatures with an rms error of $1 \mathrm{~K}$, and layer precipitable water with an rms error of 20 percent, in cases with up to 90 percent effective cloud cover. The products are designed for data assimilation purposes so as to improve numerical weather prediction, as well as for the study of climate and meteorological processes. With regard to data assimilation, one can use either the products themselves or the clear column radiances from which the products were derived.

The basic theory used to analyze AIRS/AMSU/HSB data in the presence of clouds, called the at-launch algorithm, and that used in a post-launch algorithm, which differed only in the minor details from the at launch algorithm, has been described previously ${ }^{2,3}$. The post-launch algorithm, referred to as AIRS Version $4,{ }^{3}$ had been used by the Goddard DAAC to analyze and distribute AIRS retrieval products. Susskind ${ }^{4}$ described progress towards the AIRS Version 5 retrieval algorithm. The Version 5 algorithm has since been finished and is now operational at the Goddard DAAC. The Goddard DAAC has reprocessed the entire AIRS/AMSU data set using the improved AIRS Science Team Version 5 retrieval algorithm. The AIRS Version 5 retrieval algorithm has two major improvements compared to Version 4 . The first of these improvements results from the incorporation of a new Radiative Transfer Algorithm (RTA), derived by Larrabee Strow and co-workers, that accurately accounts for the effects of Non-Local Thermodynamic Equilibrium (nonLTE) on the radiances in the $4.2 \mu \mathrm{m} \mathrm{CO}_{2}$ band. Non-LTE occurs during the day and, if not properly accounted for, makes radiances in most channels in the $4.2 \mu \mathrm{m} \mathrm{CO}_{2}$ band unusable for retrieval purposes ${ }^{3}$. Version 5 does not have this limitation. Therefore, following theoretical cloud-clearing principles ${ }^{5}$, Version 5 uses tropospheric sounding $15 \mu \mathrm{m}$ 
channels only for the purpose of deriving cloud cleared radiances $\hat{R}_{j}$ and cloud geophysical parameters. The physical retrieval step finds geophysical solutions best matching $\hat{R}_{i}$ for an ensemble of $i$ channels ${ }^{2}$. In Version 5 , most of the 4.2 $\mu \mathrm{m} \mathrm{CO}$ channels are used to determine temperature profile, as are $15 \mu \mathrm{m}$ stratospheric sounding $\mathrm{CO}_{2}$ channels which are not sensitive to clouds in the field of regard. This combination allows for the ability to produce accurate temperature profiles under more difficult cloud conditions.

The second major improvement in Version 5 is the new methodology to produce accurate case-by-case, level-by-level error estimates of retrieved temperatures at a given pressure level $\delta \mathrm{T}\left(\mathrm{p}_{\mathbf{j}}\right)$, as well as channel by channel clear column

radiance error estimates, $\delta \hat{R}_{\mathbf{i}}$. Thresholds of these error estimates are subsequently used for quality control (QC). Susskind ${ }^{6}$ showed that Version 5 produces temperature profiles of comparable accuracy to those of Version 4 , but with about twice the percentage of cases deemed acceptable in the middle troposphere, when a standard set of QC thresholds are used. Alternatively, using tighter acceptance thresholds resulted in significantly greater accuracy compared to Version 4 with about a $20 \%$ increase in acceptable profiles.

While Version 5 is a significant improvement compared to Version 4, there is still room for improvement. A further improved AIRS Science Team Version 6 algorithm is expected to be completed in late 2008. In this paper, we describe the current status (as of March 2008) of the progress made in the determination of surface skin temperature, surface spectral emissivity, and surface spectral bi-directional reflectance of solar radiation. This results in improvements not only in the retrieved surface parameters, but in other products as well. We refer to the current status system as Version $5^{+}$.

\section{CHANGES IN THE AIRS SCIENCE TEAM RETRIEVAL ALGORITHM}

The AIRS Science Team Version 5 retrieval algorithm is basically identical to that described previously ${ }^{2-4}$. The key steps of Version 5 are: 1) Start with an initial state consistent with the AIRS/AMSU observed radiance. 2) Derive IR clear column radiances $\hat{\mathrm{R}}_{i}^{0}$ valid for the $3 \times 3$ AIRS Fields of View (FOVs) within an AMSU-A Field of Regard (FOR) consistent with the observed radiances and the initial state using 58 AIRS cloud clearing channels; 3) Obtain an AIRS regression guess consistent with $\hat{R}_{i}^{0}$ using 1504 AIRS channels; 4) Derive $\hat{R}_{i}^{1}$ consistent with the AIRS radiances making use of the regression guess; 5) Derive all surface and atmospheric parameters using $\hat{R}_{\dot{1}}^{1}$ for 308 AIRS channels and AMSU radiances; 6) Derive an improved set of clear column radiances $\hat{R}_{i}^{2}$ using the AIRS physically retrieved parameters; 7) Repeat Step 5 using $\hat{R}_{i}^{2}$ to produce the final retrieval state; 8) Derive cloud parameters and OLR consistent with the solution and observed $R_{i}$;9) Apply initial quality control, which rejects the final solution if the retrieved cloud fraction is greater than $90 \%$ or other relatively coarse tests fail. In the event that retrieval is rejected, cloud parameters are determined consistent with the state used for initial cloud clearing, in conjunction with the observed AIRS radiances. Otherwise, cloud parameters are computed using the final retrieval and observed AIRS radiances, and further quality control is applied to individual geophysical parameters.

The current Version $5^{+}$retrieval system is essentially identical to that of Version 5, except for details in the physical retrieval step 5 (and 7). The physical retrieval process is comprised of a number of sequential steps listed below, each using $\hat{R}_{\mathrm{i}}$ for its own sets of channels. Geophysical parameters solved for in a given step are generally held fixed when solving for different sets of geophysical parameters in subsequent steps. In Version 5, the sequential steps are: 1) Solve for surface parameters; 2) Solve for atmospheric temperature profile $T(p) ; 3)$ Solve for atmospheric moisture profile $q(p)$; 4) Solve for ozone profile $\mathrm{O}_{3}(\mathrm{p})$; and 5) subsequently solve for $\mathrm{CO}(\mathrm{p})$ and $\mathrm{CH}_{4}(\mathrm{p})$. Version $5^{+}$adds a new step to solve for longwave surface spectral emissivity performed between step 3) and step 4).

\subsection{Version $5^{+}$changes in the surface parameter retrieval step}

Two types of changes have been made to the surface parameter retrieval step: 1) Changes to the form of the perturbation equations used to update the surface spectral emissivity $\varepsilon_{v}$ and surface spectral bi-directional reflectance $\rho_{v}$ and 2) 
changes in the channel set and emissivity and reflectivity functions being perturbed. In addition, there is a modification made to the initial guess $\rho_{v}{ }^{0}$.

\subsubsection{Changes in the form of the emissivity and reflectivity perturbation equations}

A product of the AIRS regression step is the initial IR surface spectral emissivity state $\varepsilon_{\mathrm{v}}{ }^{0}$. An initial surface bidirectional reflectance state $\rho_{v}{ }^{0}$ is generated indirectly from $\varepsilon_{v}{ }^{0}$ according to the assumption of a Lambertian surface:

$$
\rho_{v}{ }^{0}=\frac{1-\varepsilon_{v}{ }^{0}}{\pi}
$$

In Version 5, the physical surface spectral emissivity and spectral bi-directional reflectance products were determined, using $\varepsilon_{v}{ }^{0}$ and $\rho_{v}{ }^{0}$ as a first guess, according to

$$
\varepsilon_{v}=\varepsilon_{v}^{0}+\sum_{i} A_{i} F_{i}(v)
$$

and

$$
\rho_{v}=\rho_{v}{ }^{0}+\sum_{i} B_{i} G_{i}(v)
$$

where $F_{i}(v)$ and $G_{i}(v)$ are pre-assigned spectral trapezoidal perturbation functions and $A_{i}$ and $B_{i}$ are unknown coefficients solved for in the surface parameter retrieval step. One problem with use of Equation 2 in Version 5 is that occasionally, unphysical values of $\varepsilon_{v}>1$ or $\rho_{v}<0$ would occur.

This perturbation methodology has been improved in Version $5^{+}$, in which we write

$$
\left(1-\varepsilon_{v}\right)=\left(1-\varepsilon_{v}^{0}\right)\left[1+\sum A_{i} F_{i}(v)\right]
$$

and

$$
\rho_{v}=\left(\rho_{v}^{0}\right)\left[1+\sum B_{i} G_{i}(v)\right]
$$

There has also been a modification made to the initial guess $\rho_{v}{ }^{0}$ to be used in Equation $3 b$. Equation 1) does not provide an accurate initial guess for $\rho_{v}{ }^{0}$ for two reasons. The first is that the surface is usually not Lambertian. The second, and potentially more significant limitation in the use of Equation 1), is that $\rho_{v}$ is used in a term in the radiative transfer equation which multiplies the incoming solar radiation striking the earth's surface $\mathrm{H}_{v}$. In general $\mathrm{H}_{v}$ will be attenuated by clouds partially obscuring the atmospheric path from the sun to the earth's surface being observed with cloud fraction $\alpha_{\text {sun }}$. These clouds should not be confused with clouds partially obscuring the path from the surface to the satellite, whose effects are accounted for when generating $\hat{\mathbf{R}}_{\mathbf{i}}$. This unknown attenuation would result in an effective value of $\rho_{v}$ which is the true value multiplied by $\left(1-\alpha_{\text {sun }}\right)$.

Computed brightness temperatures in shortwave window channels during the day are very sensitive to $\rho_{v}$, with sensitivity increasing with frequency. An otherwise reasonable initial guess can produce values of $\hat{\Theta}_{i}-\Theta_{i}^{0}$, where $\hat{\Theta}_{i}$ is the clear column brightness temperature and $\hat{\Theta}_{i}{ }^{0}$ is the brightness temperature computed from the guess state, that differ from each other by $10 \mathrm{~K}$ or more when compared at $2448 \mathrm{~cm}^{-1}$ and $2646 \mathrm{~cm}^{-1}$. In Version $5^{+}$, we set

$$
\rho_{v}{ }^{0}=d\left(\frac{1-\varepsilon_{v}{ }^{0}}{\pi}\right)
$$

where $d$ is determined such that $\left(\hat{\Theta}_{2646}-\Theta_{2646}^{0}\right)=\left(\hat{\Theta}_{2448}-\Theta_{2448}^{0}\right)$ when $\Theta_{i}^{0}$ uses $\rho_{v}{ }^{0}$ given by Equation 4 . This constant is frequency independent. 
The form of Equation 3a resulted from the realization that the signal in $\varepsilon_{v}$ is the amount it differs from a blackbody surface, with $\varepsilon_{v}=1$. There are two practical benefits in the form of Equation 3. The first is that $\varepsilon_{v}$ is always $\leq 1$ and $\rho_{v}$ is $\geq 0$ as long as both hold for the first guess and each $A_{i}$ and $B_{i}$ is $\geq-1$. Occasionally, the physical retrieval step now results in values of $A_{i}$ or $B_{i}<-1$, in which case, they are set to -0.99 . The second benefit of equation 3 is that for surfaces expected to have spectral emissivities close to 1.0 , such as ocean and ice, the retrieved spectral emissivities will always remain close to 1.0. This stabilizes the solution.

\subsubsection{Changes in the channels and functions used to determine $T_{s}, \varepsilon_{v}$, and $\rho_{v}$}

In Version $5, T_{s}, \varepsilon_{v}$, and $\rho_{v}$ were all solved for in a single retrieval step. 15 longwave window channels between 758 $\mathrm{cm}^{-1}$ and $1228 \mathrm{~cm}^{-1}$, and 10 shortwave window channels between $2456 \mathrm{~cm}^{-1}$ and $2658 \mathrm{~cm}^{-1}$ were used simultaneously to determine $T_{s}$ along with two coefficients $A_{1}$ and $A_{2}$ of longwave spectral perturbation functions $F_{i}(v)$, one coefficient $A_{3}$ of a shortwave spectral perturbation function, and one coefficient $B_{1}$ of the spectral bi-directional reflectance perturbation function $\mathrm{G}_{1}$.

The methodology used in Version $5^{+}$represents a significant change in the philosophy in the determination of surface skin temperature $T_{s}$. Rather than use both longwave window channels (in which observations are not affected by reflected solar radiation) and shortwave window channels (which can be affected significantly by reflected solar radiation) to determine $T_{s}$, we now use only 57 shortwave window channels between $2396 \mathrm{~cm}^{-1}$ and $2660 \mathrm{~cm}^{-1}$ to determine $T_{s}$, simultaneously with coefficients of 2 shortwave spectral emissivity perturbation functions, $A_{1 s}$ and $A_{2 s}$, and 2 values of spectral bi-directional functions $B_{1}$ and $B_{2}$.

The main motivation for this modified approach is the same as that for using only shortwave $\mathrm{CO}_{2}$ absorption channels in the determination of tropospheric temperature profile. Cloud clearing errors will now result in smaller errors of $T_{s}$. This enables accurate determination of $T_{s}$ under a wider range of more difficult cloud conditions, in an analogous manner to what was found when only shortwave $\mathrm{CO}_{2}$ absorption channels were used to determine tropospheric $\mathrm{T}(\mathrm{p}$ ) (Version 5) compared to the simultaneous use of longwave and shortwave $\mathrm{CO}_{2}$ channels to determine $\mathrm{T}(\mathrm{p})$ (Version 4). ${ }^{6}$ Coefficients of 3 longwave spectral perturbation functions are now solved for, using 70 window channels between $756 \mathrm{~cm}^{-1}$ and 1234 $\mathrm{cm}^{-1}$, in a new separate step performed subsequent to the steps retrieving $T_{s}, T(p)$, and $q(p)$. Values of $T_{s}, T(p)$, and $q(p)$ are assumed known and held fixed in the determination of longwave spectral emissivity. This makes the longwave spectral emissivity retrieval step extremely stable. We expect to be able to accurately determine coefficients of more than 3 longwave emissivity functions in the future, but we have not attempted to do this yet.

This approach to use only shortwave window channel observations to determine $T_{s}$ has never been tried previously, or at least successfully utilized previously, because of concerns (or problems) in accounting for effects of reflected solar radiation during the day on the shortwave window channels. As will be shown later, results of this new approach are actually better during the day than at night.

\subsection{Changes in the temperature profile retrieval step}

The temperature profile retrieval step in Version $5^{+}$not only solves for $\mathrm{T}(\mathrm{p})$ as before, but also simultaneously updates $T_{s}, A_{1 s}, A_{2 s}, B_{1}$, and $B_{2}$. The temperature profile retrieval step now includes the 57 shortwave window channels used in the surface parameter retrieval step along with the same temperature profile retrieval channels used in Version 5.0. The temperature profile retrieval step is otherwise unchanged.

Figure 1 shows a typical AIRS cloud free brightness temperature spectrum. Channels used for different purposes are indicated in the figure. The 70 channels used to determine the coefficients of the 3 longwave emissivity perturbation functions are shown in purple. These include a channel in the center of the $\mathrm{O}_{3}$ absorption band (channels used to determine $\mathrm{O}_{3}$ profile are shown in green) which helps in the determination of the spectral emissivity in the $\mathrm{O}_{3}$ absorption region. The 57 channels used to determine surface skin temperature and shortwave values of $\varepsilon_{v}$ and $\rho_{v}$ are shown in blue. These are also used in conjunction with the red temperature sounding channels, most of which are in the spectral interval $2358 \mathrm{~cm}^{-1}-2395 \mathrm{~cm}^{-1}$. Stratospheric sounding $15 \mu \mathrm{m} \mathrm{CO} 2$ absorption channels between $660 \mathrm{~cm}^{-1}$ and $700 \mathrm{~cm}^{-1}$ 
are also included in the temperature profile retrieval step, along with some $\mathrm{N}_{2} \mathrm{O}$ absorption channels in the vicinity of $2200 \mathrm{~cm}^{-1}$. Determination of $\hat{R}_{\mathrm{i}}$ is performed using the yellow cloud clearing channels, found mostly in the $15 \mu \mathrm{m} \mathrm{CO}_{2}$ absorption region with $v_{\mathrm{i}}>700 \mathrm{~cm}^{-1}$. Also included are some longwave window channels.

\section{Sample AIRS Cloud Free Brightness Temperature}

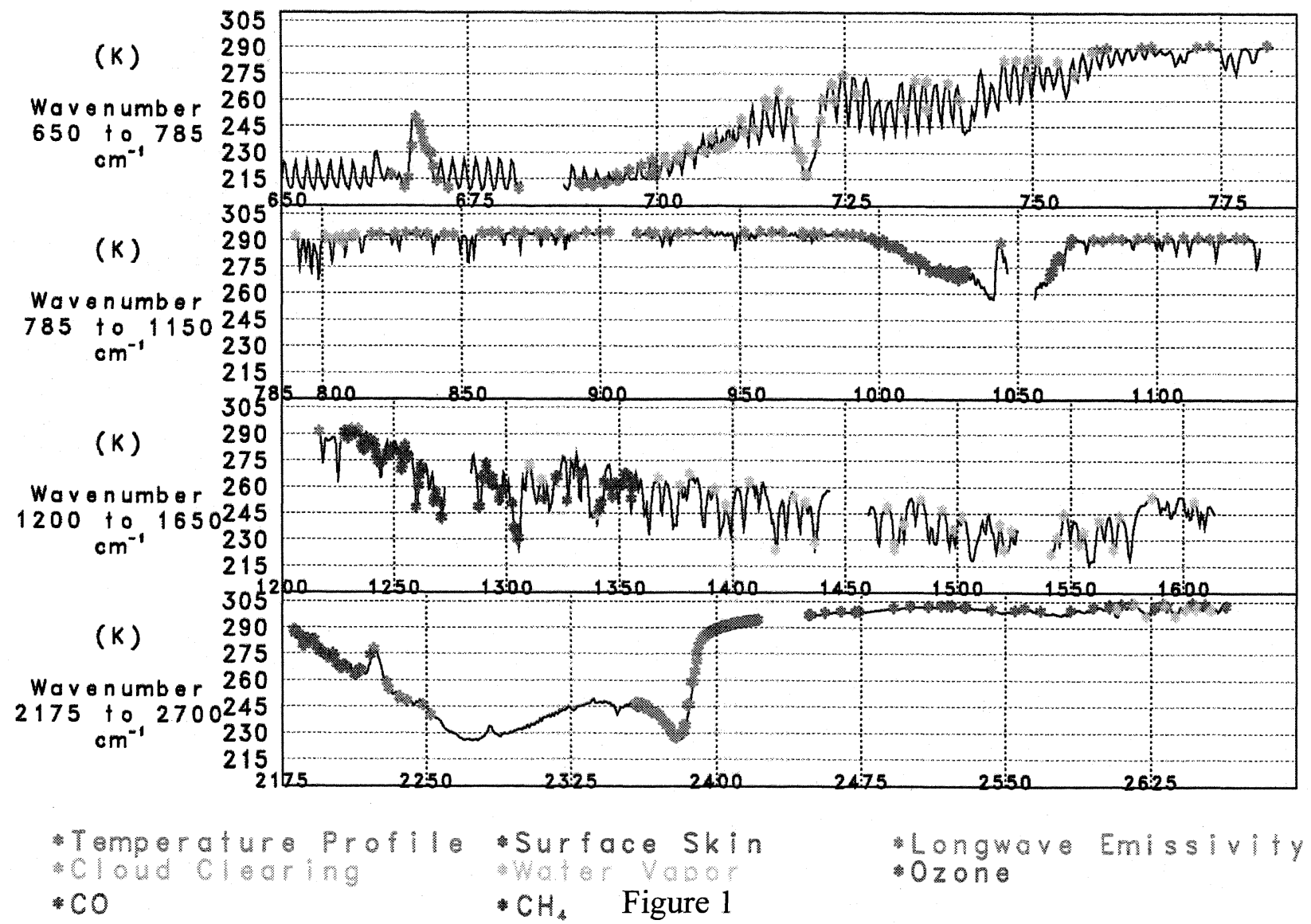

3. Improved results using Version $5^{+}$

Most of the changes made for Version $5^{+}$were in the retrieval of the surface parameters $T_{s}$ and $\varepsilon_{i}$. These have lead to improved products over land and ocean.

\subsection{Improvements over ocean}

Figure 2 a shows the Version $5^{+}$quality controlled surface skin temperature for ascending (daytime) orbits for January 25 , 2003. Grey indicates missing data resulting from orbit gaps or areas that were too cloudy to perform a quality controlled successful retrieval. Figure $2 \mathrm{c}$ shows the difference in surface skin temperature between Version $5^{+}$and Version 5 (red means Version $5^{+}$is warmer). The largest differences occur over land, where we do not have a good measure of the true values of $T_{s}$. Significant differences also occur over ocean, where we use the ECMWF SST analysis as a measure of truth. Maximum sun-glint over ocean occurs slightly left of center of the ascending orbits. Figure $2 \mathrm{c}$ shows Version $5^{+}$tends to be warmer than Version 5 to the left of the scan, and cooler to the right. Figure $2 \mathrm{~d}$ shows that $\mathrm{T}_{\mathrm{s}}$ determined using Version 5 over ocean tends to be too cold (blue) compared to ECMWF at the left of the scan, and too warm (red) at the right of the scan. Figure $2 b$ shows much better agreement with ECMWF of $T_{s}$ determined over ocean using Version $5^{+}$. 

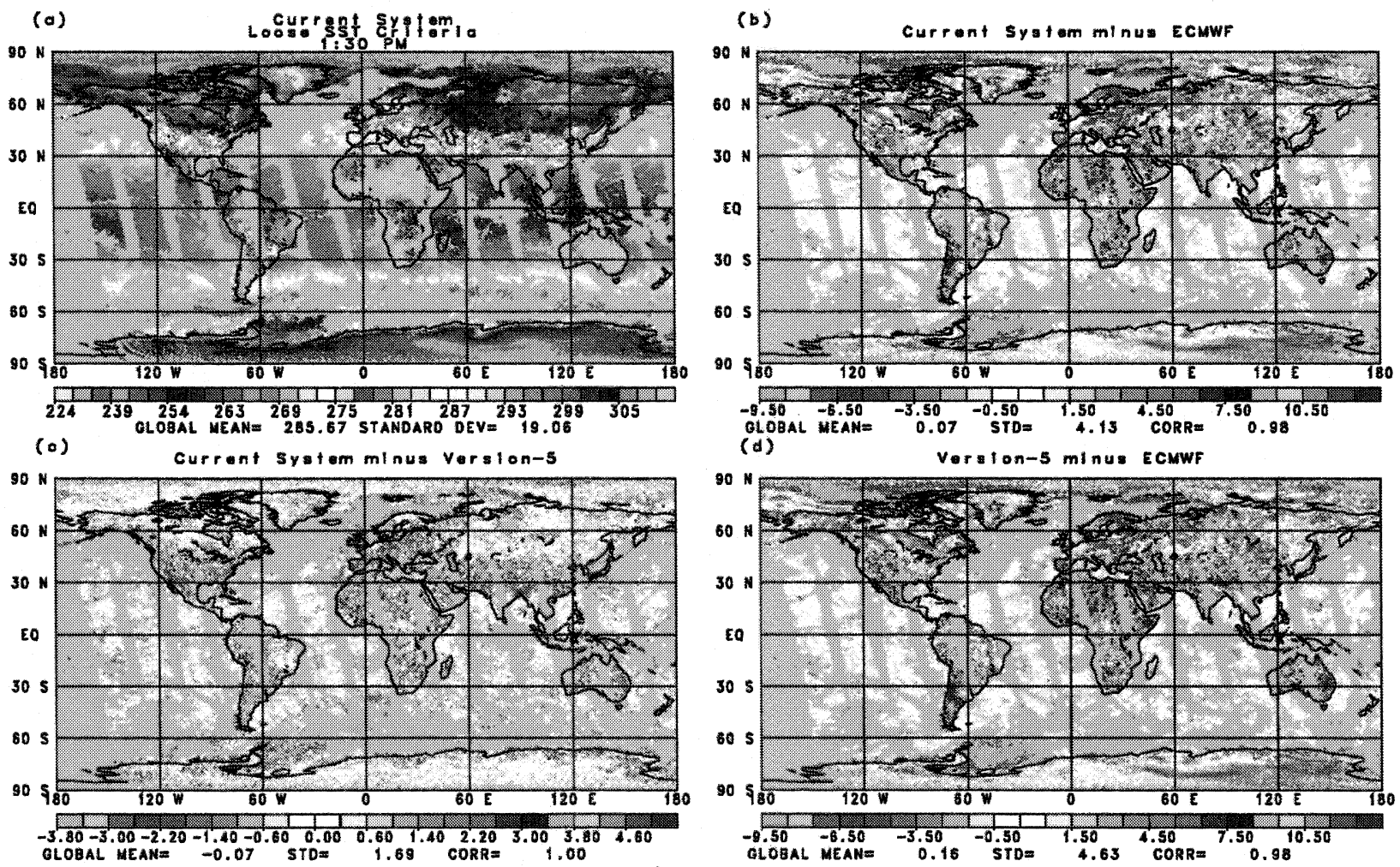

Figure 2

Surface Skin Temperature Difference from ECMWF September 6, 2002, January 25,2003, September 29, 2004

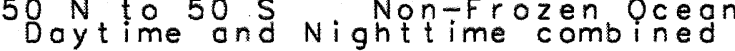

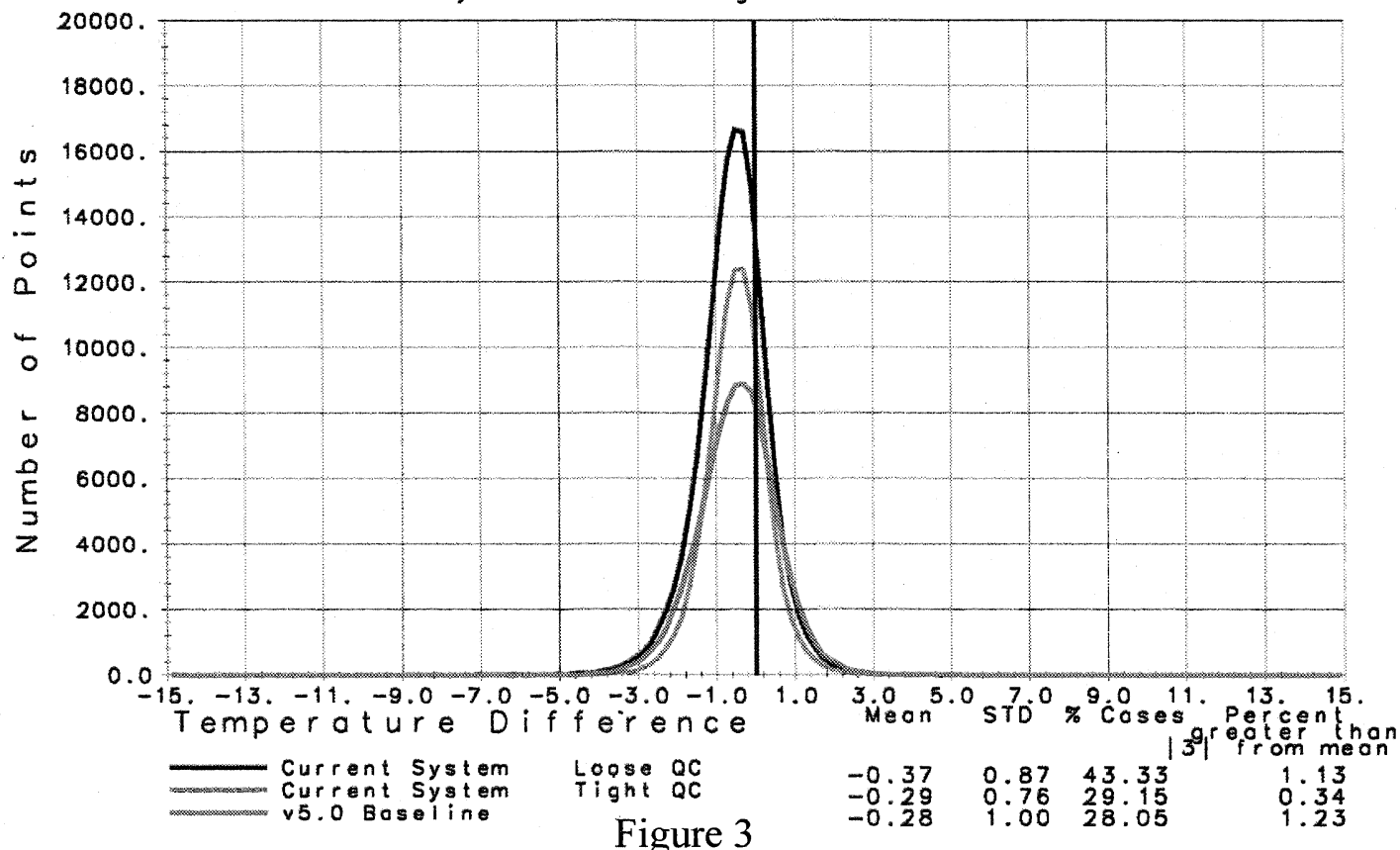


Figure 3 shows the number of quality controlled values of $T_{s}$ versus their difference from ECMWF "truth" for all accepted daytime and nighttime retrievals over ocean, $50^{\circ} \mathrm{N}-50^{\circ} \mathrm{S}$, for a composite of 3 days in which Version $5^{+}$was tested. Also shown in the mean difference from ECMWF, the standard deviation of $\left(T_{s}-E C M W F\right)$, the percent of the retrievals passing $\mathrm{QC}$, and the percent of the accepted retrievals classified as outliers, i.e., $\left|\mathrm{T}_{\mathrm{s}}-\mathrm{ECMWF}\right|>3 \mathrm{~K}$ from the mean difference. Version 5 results are shown in red. Version $5^{+}$results are shown with two different QC $\delta T_{s}$ thresholds. The tight threshold (grey) results in similar percentage yield compared to Version 5, but gives SST's with a significantly lower standard deviation from ECMWF, as well as a lesser percent of outliers. The loose QC threshold (black) provides results with a substantial increase in accepted retrievals compared to Version 5, but still with a lower standard deviation of errors and percent outliers. This considerable improvement in retrieved SST is a direct result of using shortwave channels to determine $T_{s}$, and longwave channels for cloud clearing, so as to be able to determine accurate values of $T_{s}$ under more difficult cloud conditions. Figure 2a showed the spatial coverage and accuracy of Version $5^{+} \mathrm{T}_{\mathrm{s}}$ using the Loose QC threshold.

Figures 4 and 5 give an indication of the improvement in retrieved values of $\varepsilon_{v}$ over ocean as determined by Version $5^{+}$ compared to Version 5. Ocean spectral emissivity is reasonably well described by the Masuda model ${ }^{7}$. At a given frequency, it is a small function of wind speed and a larger function of satellite zenith angle. Up to changes in wind speed, the surface emissivity over ocean should be relatively close to that predicted by the Masuda model, and not change appreciably at a given zenith angle, nor change appreciably from day to night. Figures $4 \mathrm{a}$ and $4 \mathrm{~b}$ show the angular dependence of the 3 day mean of the difference of retrieved values of $\varepsilon_{v}$ from the Masuda model for $950 \mathrm{~cm}^{-1}$ and $2400 \mathrm{~cm}^{-1}$ respectively. Version $5^{+}$values are in black for AM cases and grey for PM cases, while Version 5 values

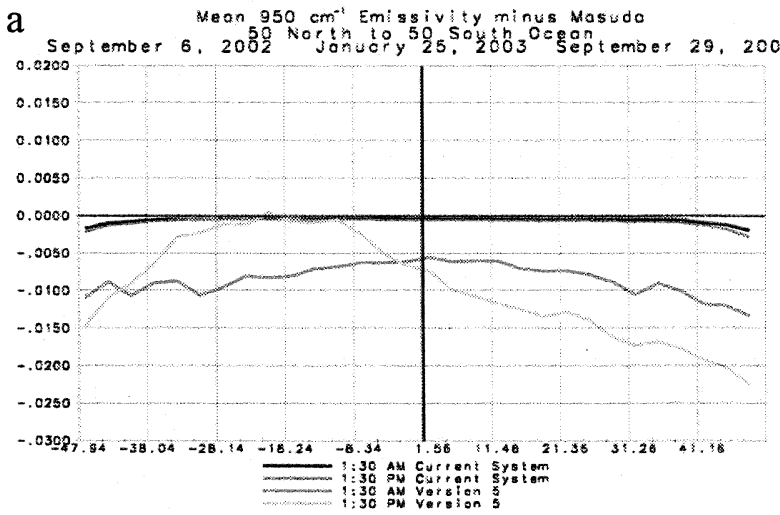

c
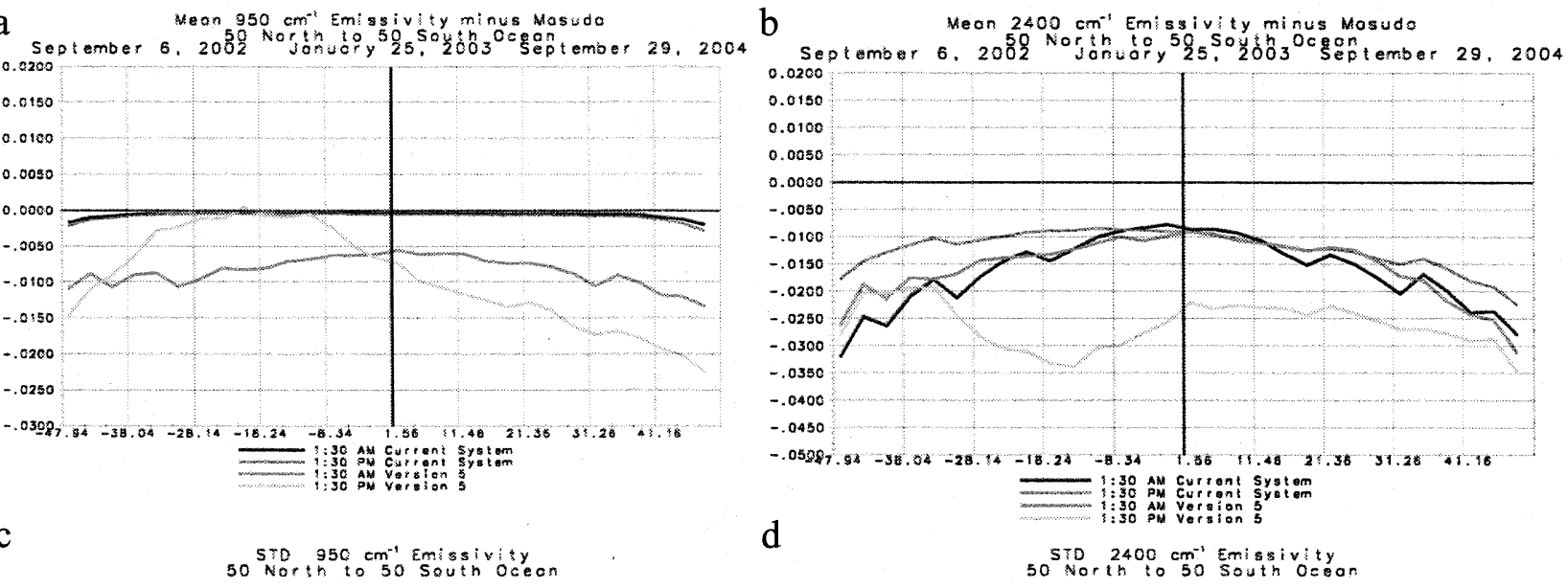

$-.05287 .54-58.04-28.54-18.24-8.34$.

d
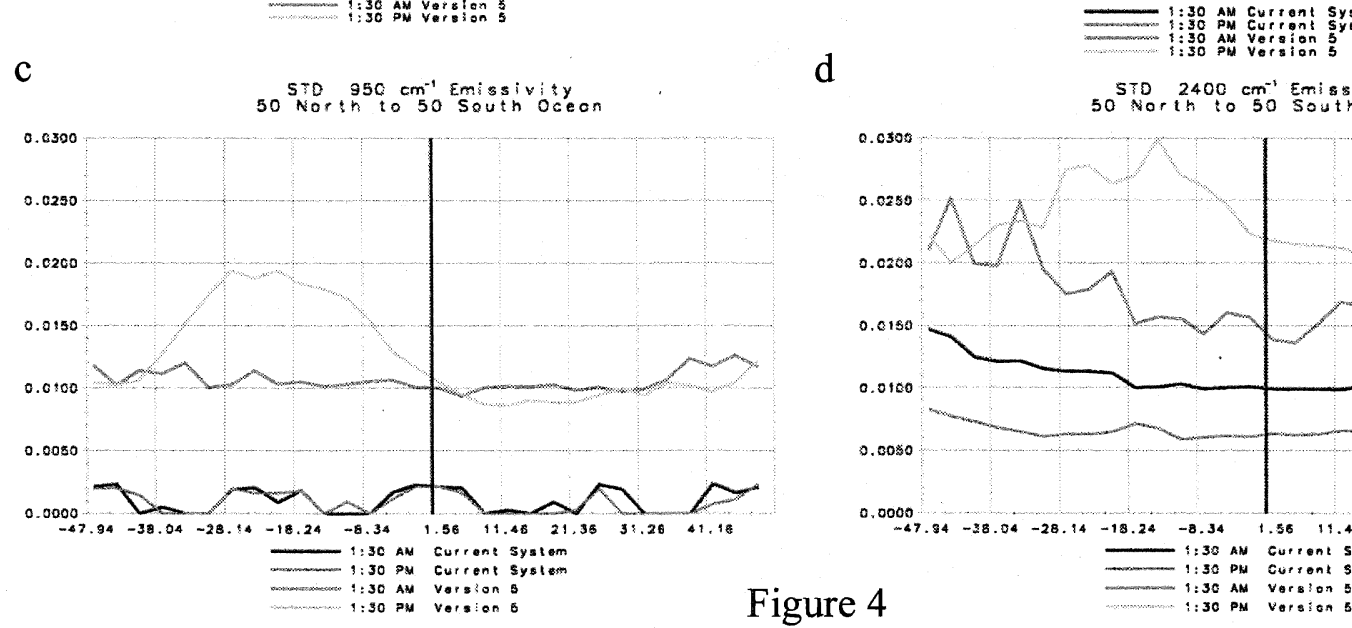

Figure 4

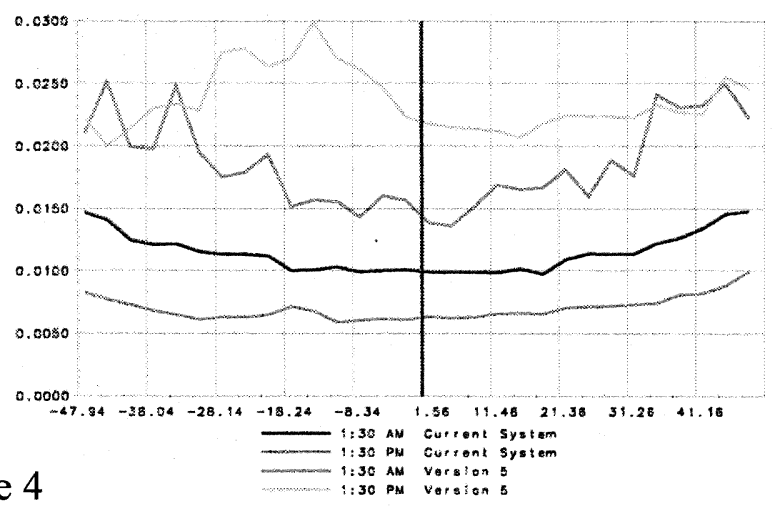


are in dark blue and light blue for AM and PM cases respectively. Maximum sun-glint occurs in the vicinity of $-20^{\circ}$ satellite zenith angle. Figures $4 \mathrm{c}$ and $4 \mathrm{~d}$ show analogous results for the standard deviation of retrieved values of $\varepsilon_{\mathrm{v}}$ at a given satellite zenith angle. It is apparent that the mean retrieved values of $\varepsilon$ over ocean at $950 \mathrm{~cm}^{-1}$ and $2400 \mathrm{~cm}^{-1}$ are considerably closer to the Masuda model, both day and night, using Version $5^{+}$compared to Version 5 . In addition they are also much more stable, having a considerably lower standard deviation day and night. It is informative to see that Version 5 standard deviations of $\varepsilon_{v}$ are larger during the day than at night, especially in the sun-glint area $\left(\approx-20^{\circ}\right)$. In Version $5^{+}$, the standard deviations are very small day and night at $950 \mathrm{~cm}^{-1}$ and are actually lower during the day than at night at $2400 \mathrm{~cm}^{-1}$, with no indication of instabilities as a result of sun-glint.

Figure 5 shows the mean difference of $\mathrm{AM}$ and PM retrieved surface emissivities for four frequencies. These AM/PM differences are considerably smaller in Version $5^{+}$than in Version 5.
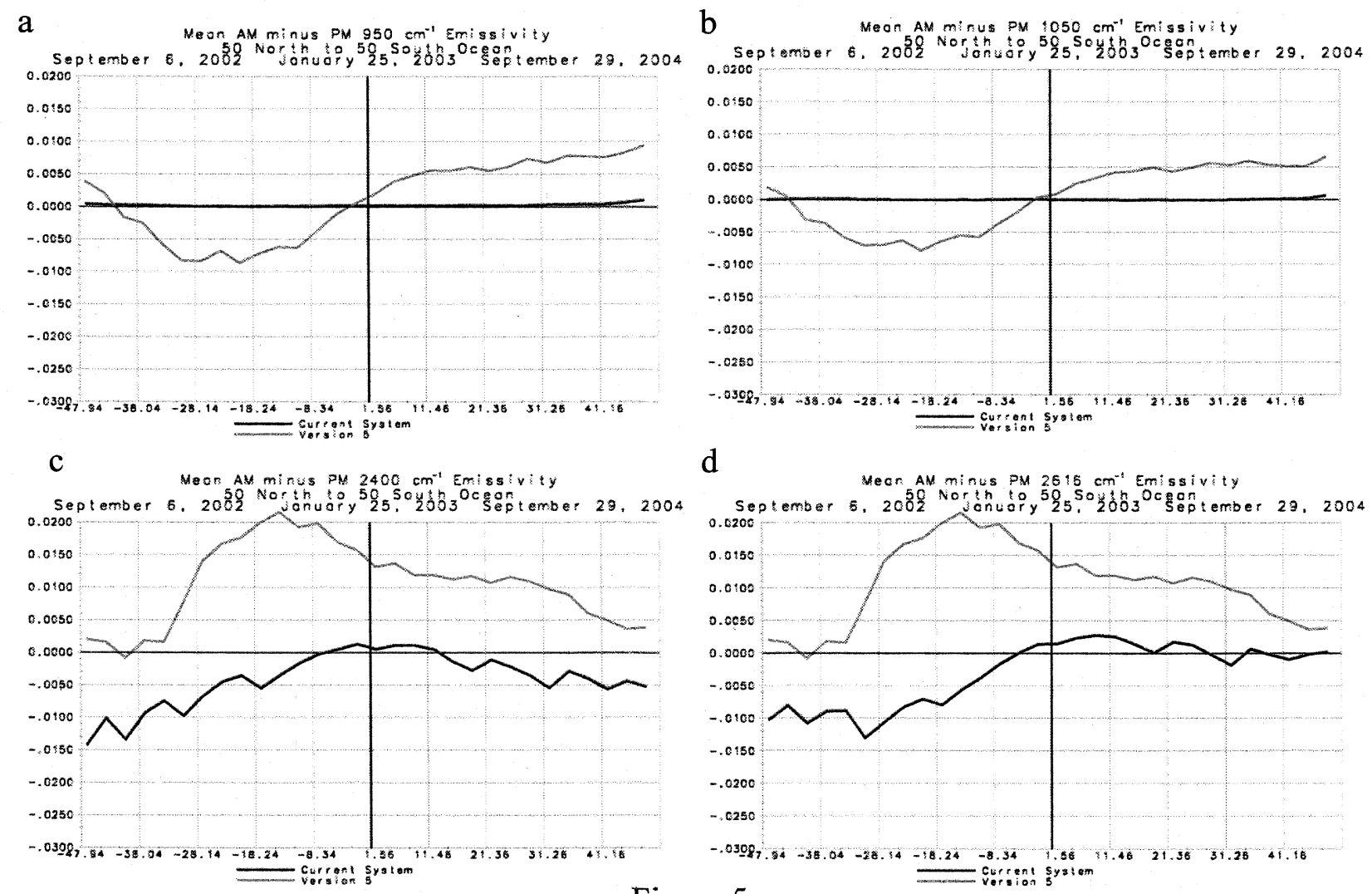

$\mathrm{d}$

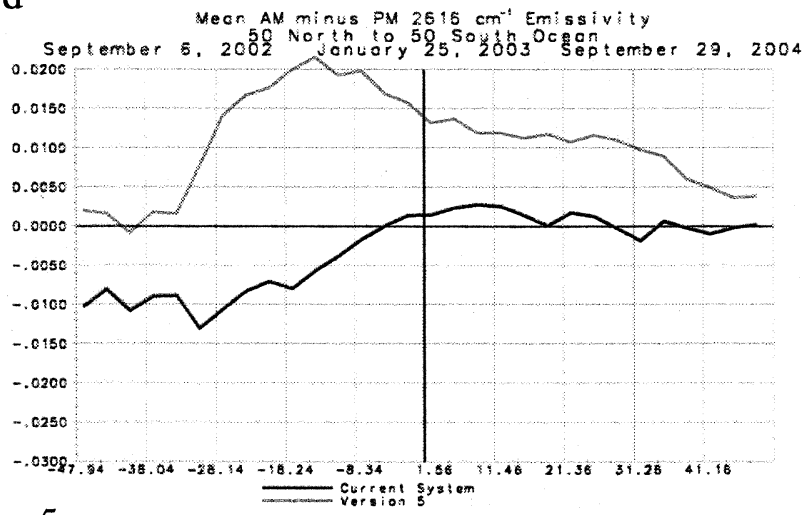

Figure 5

\subsection{Improvements over land}

Unlike ocean where both surface skin temperature and surface spectral emissivity are reasonably well known, there is no good measure of truth over land for either quantity. Therefore, we use indirect means to evaluate any improvements in retrieved surface parameters. While we do not have a good measure of surface spectral emissivity at a given location, we know it should not change appreciably from day to night. Figure 6 shows mean differences of retrieved surface emissivity as a function of zenith angle over land. Day/night differences of retrieved emissivity using Version $5^{+}$over land are considerably lower than they were using Version 5. This result is analogous to what was found over ocean (see Figure 5). 
a Nean AM minius PM $950 \mathrm{~cm}^{-1}$ Emissivily

b Meon AM minus PM $1050 \mathrm{~cm}^{-1}$ Emissivity

b Meon AM minus PM $1050 \mathrm{~cm}^{-1}$ Emissivity

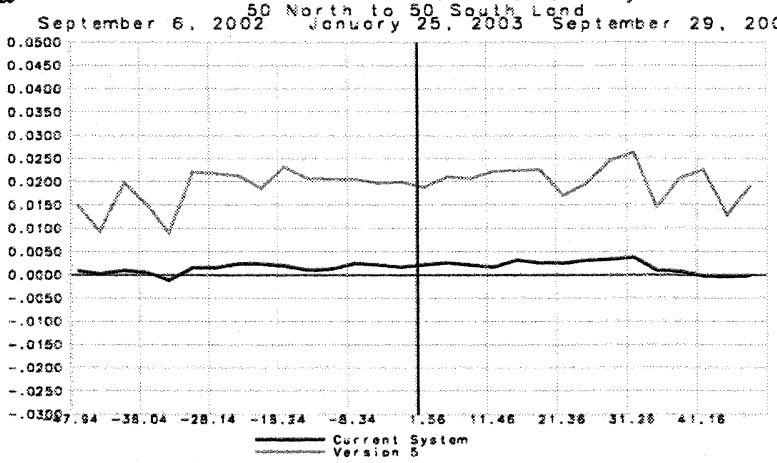

C

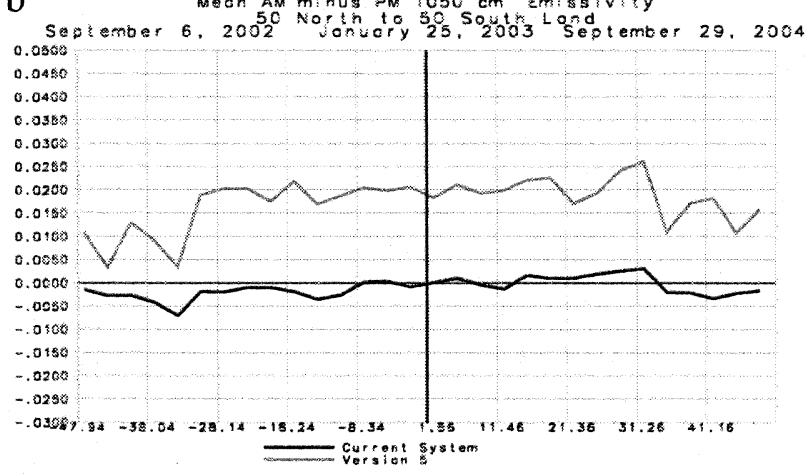

d

Meon AM mirus PM $2400 \mathrm{~cm}^{-1} \mathrm{Em}$ issivity

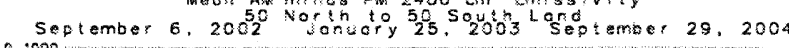

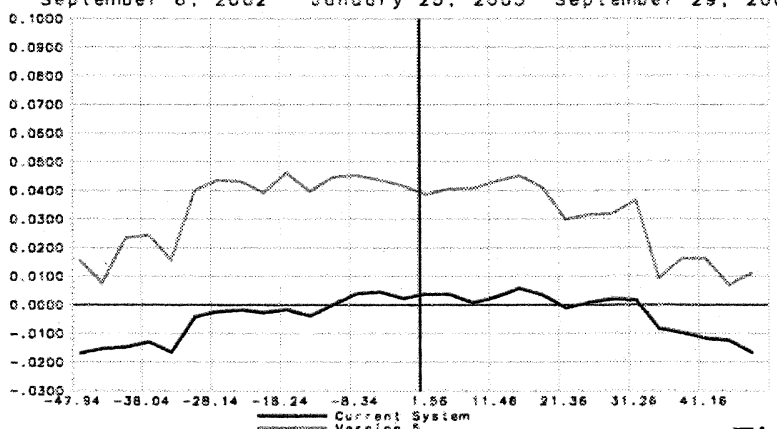

d

Mech AM minus PM $2616 \mathrm{~cm}^{-3}$ Emissivity

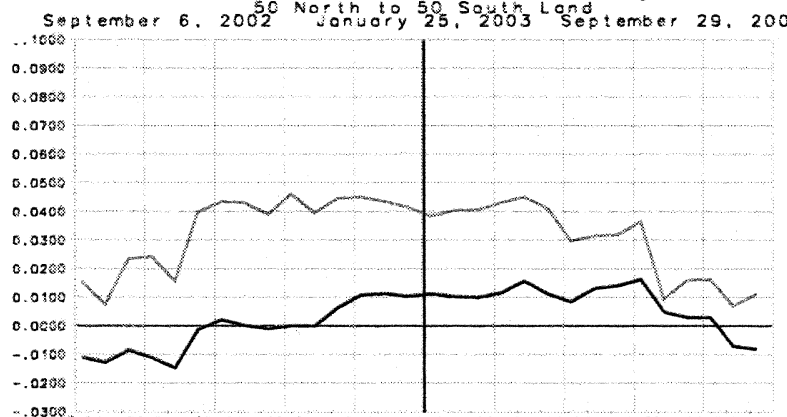

Figure 6

Septomber 6, 2002, Janugiry, 25, 2003itand september 29, 2004

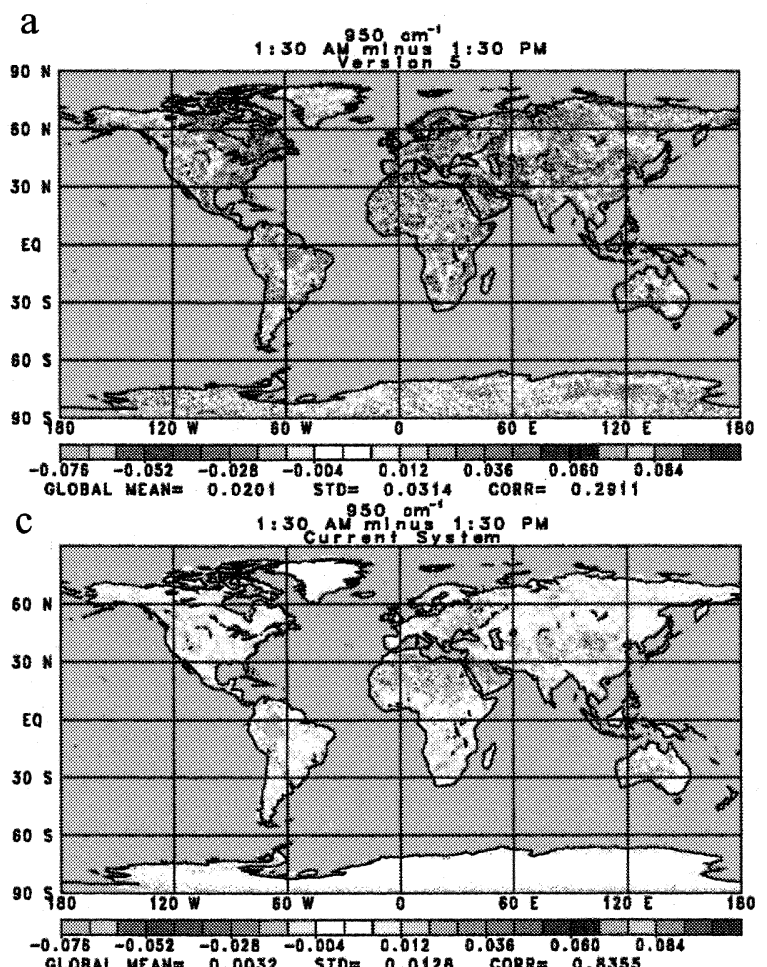

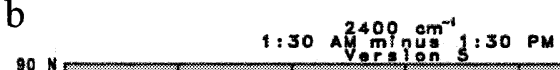
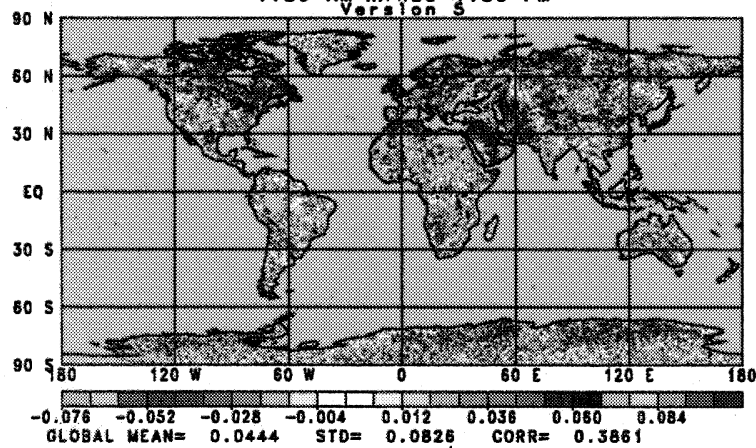

d

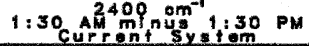

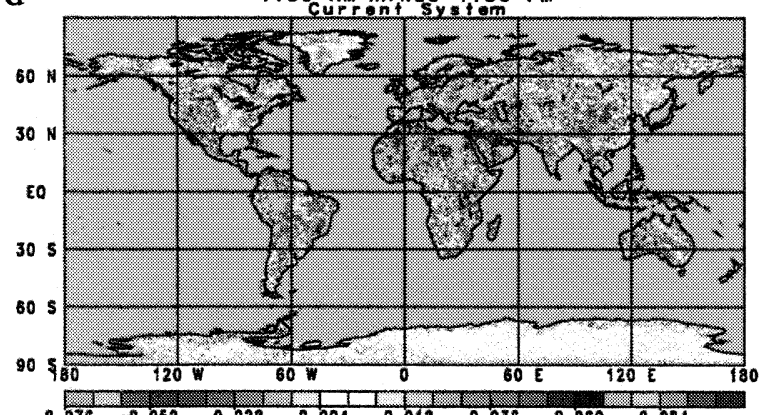

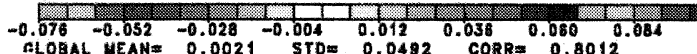

Figure 7 
Figure 7 shows the spatial distribution of the AM/PM differences of retrieved emissivity over land at $950 \mathrm{~cm}^{-1}$ and 2400 $\mathrm{cm}^{-1}$. These differences are much smaller in Version $5^{+}$(Figure $7 \mathrm{c}$ and 7d) than in Version 5 (Figures 7a and 7b), especially over ice. The spatial correlation of the day and night emissivity maps are given in the figure, and are much higher at both frequencies in Version $5^{+}(\approx 0.8)$ than in Version $5(\approx 0.3)$.

Figure 8a and $8 \mathrm{~b}$ shows RMS $1 \mathrm{~km}$ layer mean differences of quality controlled day and night oceanic and land temperature profile retrievals from a colocated ECMWF analysis determined using Version 5 (red) and Version $5^{+}$ (grey). It is apparent that the improvement in retrieved surface parameters has also improved the accuracy of the quality controlled temperature profile by about $0.1 \mathrm{~K}$ in the lowest $2 \mathrm{~km}$ of the atmosphere over ocean, and by $0.2 \mathrm{~K}$ over land.

a

LAYER VEAN RNS TENPERATURE $\left({ }^{\circ} \mathrm{C}\right)$

Dlforences from ECWWF

Sep 5, 2002, Januery 25, 2003, ond Sep 29, 200 $50 \mathrm{~N}$ to $50 \mathrm{~S}$ Ocear

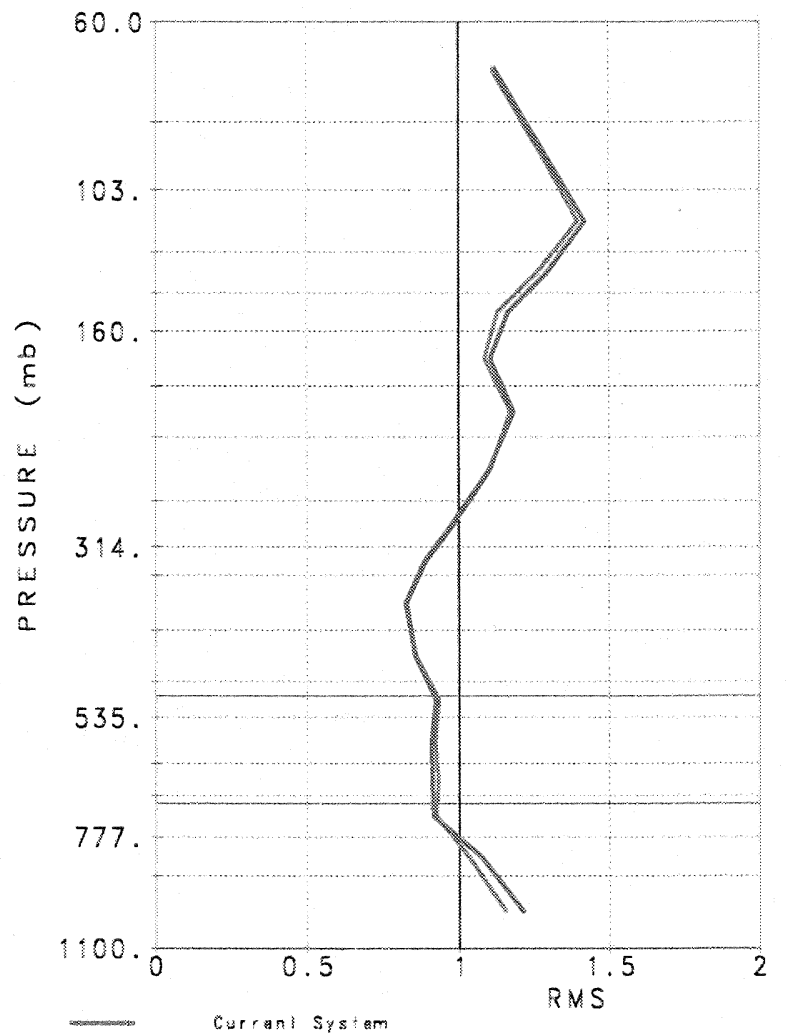

b
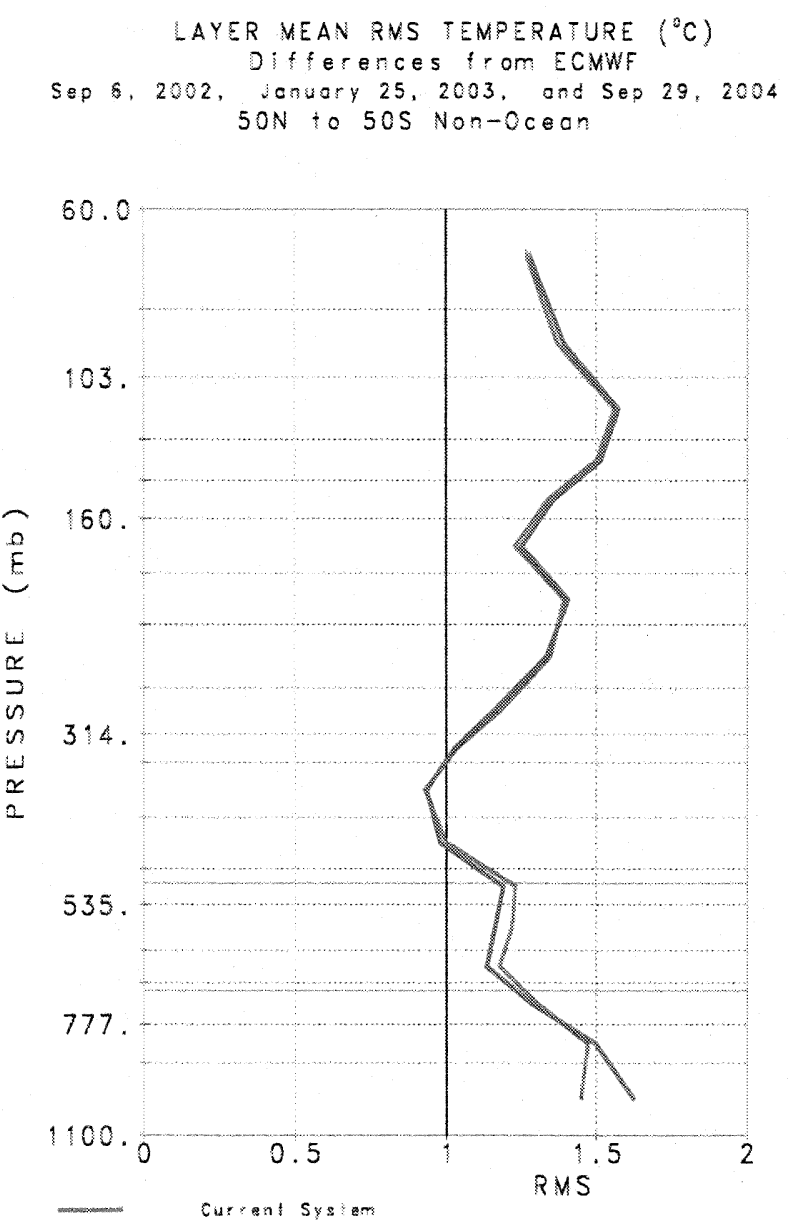

Curent sys an

Versions 5

Figure 8 
OZONE (DU)

January 25,2003
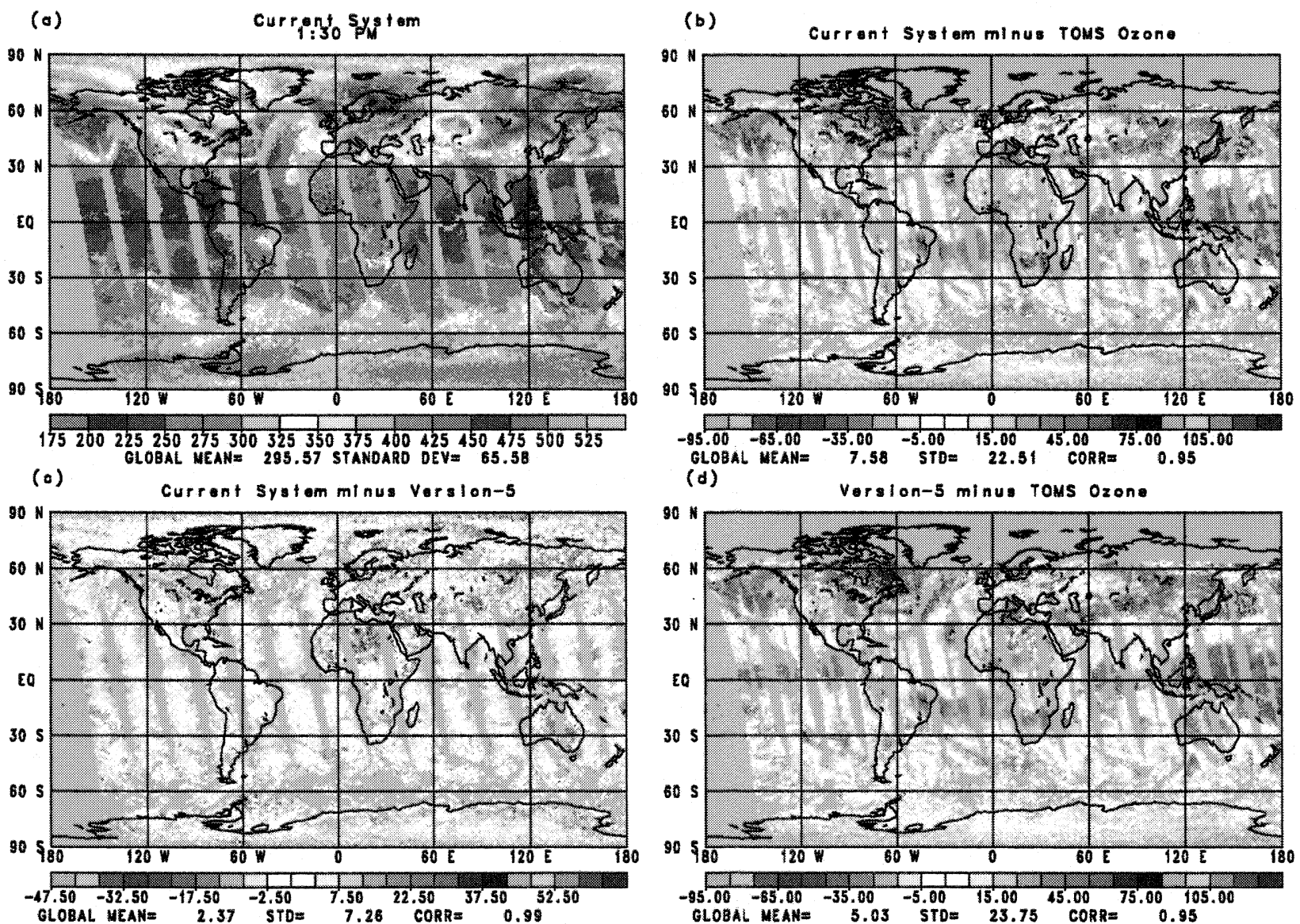

Figure 9

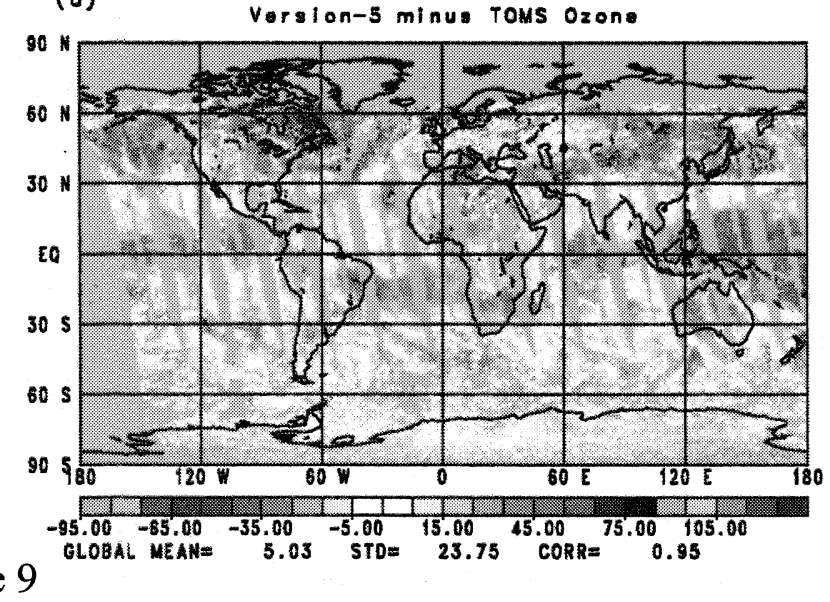

Figure 9 gives an indication of the improvement in total ozone, as compared to colocated TOMS total ozone, resulting from the changes made in Version $5^{+}$. Figure 9a shows the quality controlled AIRS Version $5^{+}$total $\mathrm{O}_{3}$ field derived for daytime orbits on January 25, 2003. Figure 9c shows Version $5^{+}$total ozone minus Version 5 total $\mathrm{O}_{3}$ (red means Version $5^{+}$is higher). Total $\mathrm{O}_{3}$ retrieved over ocean has increased compared to Version 5 , especially at the end of the scan. Figure 9d shows that Version 5 total $\mathrm{O}_{3}$ tended to be low compared to TOMS over ocean. Figure $9 \mathrm{~b}$ shows agreement of total $\mathrm{O}_{3}$ with TOMS is now improved over ocean.

Over Northern Hemisphere extra-tropical land, total $\mathrm{O}_{3}$ retrieved using Version $5^{+}$is generally lower than that retrieved using Version 5. In the central Sahara desert, total $\mathrm{O}_{3}$ is now significantly higher than it was in Version 5. All of these changes are in the correct direction so as to decrease differences from TOMS of Version $5^{+}$total $\mathrm{O}_{3}$ over land compared to Version 5 total $\mathrm{O}_{3}$. Note that no changes have been made to the $\mathrm{O}_{3}$ profile retrieval step, so the improvements are an indirect result of improvements in skin temperature and surface emissivity near $1050 \mathrm{~cm}^{-1}$. 


\section{Summary}

Improved methodology has been developed to determine surface skin temperature $T_{s}$ and surface spectral emissivity $\varepsilon_{v}$ from AIRS/AMSU observations under partial cloud cover. In this methodology, only shortwave window channels are used to simultaneously determine $T_{s}$ and shortwave values of $\varepsilon_{v}$, along with shortwave values of effective surface bidirectional reflectance of solar radiation $\rho_{v}$. Longwave values of $\varepsilon_{v}$ are determined in a subsequent step using only longwave window channels, consistent with the previously derived value of $T_{s}$. This results in considerable improvement in retrieved surface parameters compared to what was obtained using Version 5, both day and night. It also allows for very accurate soundings of SST under more difficult cloud conditions compared to the methodology used in Version 5. The improvement in retrieved surface parameters also resulted in improved values of quality controlled boundary layer temperatures as well as of total $\mathrm{O}_{3}$. This research is being conducted as part of the development of the new AIRS Science Team Version 6 retrieval algorithm which is expected to be completed in late 2008 and used by the Goddard DAAC starting in 2009.

\section{REFERENCES}

1. T.S., Pagano, H.H. Aumann, D.E. Hagan, and K. Overoye, "Prelaunch and in-flight radiometric calibration of the Atmospheric Infrared Sounder (AIRS)," IEEE Trans. Geosci. Remote Sensing, 41, 265-273, 2003.

2. J. Susskind, C.D. Barnet, and J.M. Blaisdell, "Retrieval of atmospheric and surface parameters from AIRS/AMSU/HSB data in the presence of clouds," IEEE Trans. Geosci. Remote Sensing, 41, 390-409, 2003.

3. J. Susskind, C. Barnet, J. Blaisdell, L. Iredell, F. Keita, L. Kouvaris, G. Molnar, and M. Chahine, "Accuracy of geophysical parameters derived from Atmospheric Infrared Sounder/Advanced Microwave Sounding Unit as a function of fractional cloud cover," J. Geophys. Res., 111,D09S17, doi:10.1029/2005JD006272, 2006.

4. J. Susskind, "Recent theoretical advances in analysis of AIRS/AMSU sounding data," Proc. of SPIE Vol. 6565, Algorithms and Technologies for Multispectral, Hyperspectral, and Ultraspectral Imagery XIII, Orlando, FL, April 9-12, 2007, 65651H, 0277-786X/07/\$18,doi:10.1117/12.718448, 65651H-1 - 65651H-12, 2007.

5. M.T. Chahine, "Remote Sounding of Cloudy Atmospheres. I. The Single Cloud Layer," J. Atmos. Sci., 3, 233-243, 1974.

6. J. Susskind, "Improved Atmospheric Soundings and Error Estimates from Analysis of AIRS/AMSU Data," Proc. of SPIE Vol. 6684, Atmospheric and Environmental Remote Sensing Data Processing and Utilization III: Readiness for GEOSS, San Diego, CA, August 26-31, 2007.

7. K. Masuda, T. Takashima, and Y. Takayama, "Emissivity of pure and sea waters for the model sea surface in the infrared window region," Remote Sens. Environ., 24, 313-329, 1998. 